\title{
Make Unification on $\alpha$-admissible Mappings and Related Fixed Point Theorems
}

\author{
A. H. Ansari, S. Chandok
}

\begin{abstract}
In this paper, we introduced the concepts pair $(\mathscr{F}, h)$ a upclass of type $I I$ and $\alpha_{\beta^{-}}$ contractive mappings and show that theorems in [4]reduce to corollaries in this paper. that is, all them can obtain of one theorem. in end we state example for support main result.
\end{abstract}

Keywords: $\alpha$-admissible mappings, contractive mappings, $\alpha_{\beta}$-contractive, fixed point.

\section{Introduction and mathematical preliminaries}

Fixed point theory has gained very large impetus due its wide range of applications in various fields such as engineering, economics, computer science, and many others. It is well known that the contractive-type conditions are very indispensable in the study of fixed point theory and Banach's fixed point theorem [1] for contraction mappings is one of the pivotal result in analysis. This theorem that has been extended and generalized by various authors (see, e.g., [4]-[9]) and has many applications in mathematics and other related disciplines as well.

In an attempt to generalize the Banach contraction principle, many researchers extended the following result in certain directions.

Theorem 1 (see, $[6,7,8])$ Let $(X, d)$ be a complete metric space and $T: X \rightarrow X$ be a mapping. Assume that there exists a function $\beta:[0, \infty) \rightarrow[0,1]$ such that, for any bounded sequence $\left\{t_{n}\right\}$ of positive reals, $\beta\left(t_{n}\right) \rightarrow 1$ implies $t_{n} \rightarrow 0$ and $d(T x, T y) \leq \beta(d(x, y)) d(x, y)$ for all $x, y \in X$. Then $T$ has a unique fixed point.

In this paper, we introduced the concepts pair $(\mathscr{F}, h)$ a upper class of type $I I$ and $\alpha_{\beta}$ contractive mappings to show that theorems in [4] reduce to corollaries in this paper. That is, all them can obtain of main theorem. in end we state example for support main result.

Manuscript received October 29, 2015; accepted March 2, 2016.

A. H. Ansari is with the Department of Mathematics, Karaj Branch, Islamic Azad University, Karaj, Iran, S.Chandok is with the School of Mathematics Thapar University Patiala 147004 Punjab India 
To start with we give some notations and introduce some definitions which will be used in the sequel.

In 2014 the concept of pair $(\mathscr{F}, h)$ is an upper class was introduced by A. H. Ansari in [2] that then reform definition(part words ) it in [3]

Definition 1 [2],[3] We say that the function $h: \mathbb{R}^{+} \times \mathbb{R}^{+} \times \mathbb{R}^{+} \rightarrow \mathbb{R}$ is a function of subclass of type II, if $x, y \geq 1 \Longrightarrow h(1,1, z) \leq h(x, y, z)$ for all $z \in \mathbb{R}^{+}$.

Example 1 [2],[3]Define $h: \mathbb{R}^{+} \times \mathbb{R}^{+} \times \mathbb{R}^{+} \rightarrow \mathbb{R}$ by:

(a) $h(x, y, z)=(z+l)^{x y}, l>1$;

(b) $h(x, y, z)=(x y+l)^{z}, l>1$;

(c) $h(x, y, z)=z$;

(d) $h(x, y, z)=x^{m} y^{n} z^{p}, m, n, p \in \mathbb{N}$;

(e) $h(x, y, z)=\frac{x^{m}+x^{n} y^{p}+y^{q}}{3} z^{k}, m, n, p, q, k \in \mathbb{N}$

for all $x, y, z \in \mathbb{R}^{+}$. Then $h$ is a function of subclass of type II.

Definition 2 [2],[3]Let $h: \mathbb{R}^{+} \times \mathbb{R}^{+} \times \mathbb{R}^{+} \rightarrow \mathbb{R}$ and $\mathscr{F}: \mathbb{R}^{+} \times \mathbb{R}^{+} \rightarrow \mathbb{R}$, then we say that the pair $(\mathscr{F}, h)$ is an upper class of type II, if $h$ is a subclass of type II and: (i) $0 \leq s \leq$ $1 \Longrightarrow \mathscr{F}(s, t) \leq \mathscr{F}(1, t)$, (ii) $h(1,1, z) \leq \mathscr{F}(s, t) \Longrightarrow z \leq$ st for all $s, t, z \in \mathbb{R}^{+}$.

Example 2 [2],[3]Define $h: \mathbb{R}^{+} \times \mathbb{R}^{+} \times \mathbb{R}^{+} \rightarrow \mathbb{R}$ and $\mathscr{F}: \mathbb{R}^{+} \times \mathbb{R}^{+} \rightarrow \mathbb{R}$ by:

(a) $h(x, y, z)=(z+l)^{x y}, l>1, \mathscr{F}\{(\mathrm{f} s, t)=s t+l$;

(b) $h(x, y, z)=(x y+l)^{z}, l>1, \mathscr{F}(s, t)=(1+l)^{s t}$;

(c) $h(x, y, z)=z, F(s, t)=s t$;

(d) $h(x, y, z)=x^{m} y^{n} z^{p}, m, n, p \in \mathbb{N}, \mathscr{F}(s, t)=s^{p} t^{p}$

(e) $h(x, y, z)=\frac{x^{m}+x^{n} y^{p}+y^{q}}{3} z^{k}, m, n, p, q, k \in \mathbb{N}, \mathscr{F}(s, t)=s^{k} t^{k}$

for all $x, y, z, s, t \in \mathbb{R}^{+}$. Then the pair $(\mathscr{F}, h)$ is an upper class of type II.

Definition 3 Let $(X, d)$ be a metric space and $T: X \rightarrow X$, a nonempty subset $F$ of $X$ is valled invariant under the $T$ if $T x \in F$ for every $x \in F$.

Definition 4 Let $T: X \rightarrow X$ and $\alpha: F \times F \rightarrow \mathbb{R}^{+}$. We say that $T$ is an $\alpha_{F}$-admissible mapping if $\alpha(x, y) \geq 1$ implies $\alpha(T x, T y) \geq 1, x, y \in F$. 
Note: A mapping $T$ is called an $\alpha$-admissible mapping (see [9]) if we take $F=X$ in Definition 4.

Definition 5 A function $\psi:[0, \infty) \rightarrow[0, \infty)$ is called altering distance function if the following properties are satisfied:

1. $\psi$ is continuous and non-decreasing;

2. $\psi^{-1}(\{0\})=0$.

We denote by $\Psi$ the set of all altering distance functions.

Lemma 1 [10] Suppose $(X, d)$ is a metric space. Let $\left\{x_{n}\right\}$ be a sequence in $X$ such that $d\left(x_{n}, x_{n+1}\right) \rightarrow 0$ as $n \rightarrow \infty$. If $\left\{x_{n}\right\}$ is not a Cauchy sequence then there exist an $\varepsilon>0$ and sequences of positive integers $\{m(k)\}$ and $\{n(k)\}$ with

$m(k)>n(k)>k$ such that $d\left(x_{m(k)}, x_{n(k)}\right) \geq \varepsilon, d\left(x_{m(k)-1}, x_{n(k)}\right)<\varepsilon$ and

(i) $\lim _{k \rightarrow \infty} d\left(x_{m(k)-1}, x_{n(k)+1}\right)=\varepsilon$;

(ii) $\lim _{k \rightarrow \infty} d\left(x_{m(k)}, x_{n(k)}\right)=\varepsilon$;

(iii). $\lim _{k \rightarrow \infty} d\left(x_{m(k)-1}, x_{n(k)}\right)=\varepsilon$

Note that also we have that $\lim _{k \rightarrow \infty} d\left(x_{m(k)+1}, x_{n(k)+1}\right)=\varepsilon$;

\section{Main Results}

Definition 6 Let $(X, d)$ be a metric space, $F$ a nonempty subset of $X, T: X \rightarrow X$ and $\alpha: F \times F \rightarrow \mathbb{R}^{+}$. A mapping $T$ is said to be $\alpha_{\beta}$-contractive mapping if there exists a $\beta:[0, \infty) \rightarrow[0,1]$ such that for any bounded sequence $\left\{t_{n}\right\}$ of positive reals, $\beta\left(t_{n}\right) \rightarrow 1$ implies $t_{n} \rightarrow 0$, such that for all $x, y \in F$, following condition holds:

$$
h(\alpha(x, T x), \alpha(y, T y), \psi d(T x, T y)) \leq \mathscr{F}(\beta(d(x, y)), \psi d(x, y)),
$$

Where pair $(\mathscr{F}, h)$ is a upclass of type II and $\psi \in \Psi$.

Theorem 2 Let $(X, d)$ be a complete metric space, $F$ a nonempty closed subset of $X, T$ : $X \rightarrow X$ is an $\alpha_{F}$-admissible mapping and $F$ is invariant under $T$. Further assume that $T$ is an $\alpha_{\beta}$-contractive mapping. Suppose that there exists $x_{0} \in F$ such that $\alpha\left(x_{0}, T x_{0}\right) \geq 1$ and either of the following conditions hold:

(a) $T$ is continuous, or

(b) if $\left\{x_{n}\right\}$ is a sequence in $F$ such that $x_{n} \rightarrow x, \alpha\left(x_{n}, x_{n+1}\right) \geq 1$, for all $n$, then $\alpha(x, T x) \geq 1$.

Then $T$ has a fixed point. 
Proof Let $x_{0} \in F$ such that $\alpha\left(x_{0}, T x_{0}\right) \geq 1$.Now,we construct a sequence $\left\{x_{n}\right\}$ in $F$ by $x_{n}=T x_{n-1}$, for $n \geq 1$, such that $\alpha\left(x_{n}, x_{n+1}\right)=\alpha\left(x_{n}, T x_{n}\right) \geq 1$. Substituting $x=x_{n-1}$ and $y=x_{n}$ in (1) , we obtain

$$
\begin{aligned}
h\left(1,1, \psi\left(d\left(x_{n}, x_{n+1}\right)\right)\right) & \leq h\left(\alpha\left(x_{n-1}, x_{n}\right), \alpha\left(x_{n}, x_{n+1}\right), \psi\left(d\left(x_{n}, x_{n+1}\right)\right)\right) \\
& \leq \mathscr{F}\left(\beta\left(d\left(x_{n-1}, x_{n}\right)\right), \psi\left(d\left(x_{n-1}, x_{n}\right)\right)\right)
\end{aligned}
$$

which implies that

$$
\begin{aligned}
\psi\left(d\left(x_{n}, x_{n+1}\right)\right) & \leq \beta\left(d\left(x_{n-1}, x_{n}\right)\right) \psi\left(d\left(x_{n-1}, x_{n}\right)\right) \\
& \leq \psi\left(d\left(x_{n-1}, x_{n}\right)\right) .
\end{aligned}
$$

As $\psi \in \Psi$,

$$
d\left(x_{n}, x_{n+1}\right) \leq d\left(x_{n-1}, x_{n}\right),
$$

for every $n \in \mathbb{N}$, the sequence $\left\{d\left(x_{n}, x_{n+1}\right)\right\}$ is decreasing so that for the nonnegative decreasing sequence $\left\{d\left(x_{n}, x_{n+1}\right)\right\}$, there exists some $r \geq 0$, such that

$$
\lim _{n \rightarrow \infty} d\left(x_{n}, x_{n+1}\right)=r
$$

Further from (2), it implies that

$$
\frac{\psi\left(d\left(x_{n}, x_{n+1}\right)\right)}{\psi\left(d\left(x_{n-1}, x_{n}\right)\right)} \leq \beta\left(d\left(x_{n-1}, x_{n}\right)\right) \leq 1 .
$$

On letting $n \rightarrow \infty$ in above inequality, we have $\lim _{n \rightarrow \infty} \beta\left(d\left(x_{n-1}, x_{n}\right)\right)=1$, and this implies that

$$
\lim _{n \rightarrow \infty} d\left(x_{n}, x_{n+1}\right)=0 .
$$

Now, we will show that $\left\{x_{n}\right\}$ is a Cauchy sequence. Suppose, to the contrary, that $\left\{x_{n}\right\}$ is not a Cauchy sequence.

By lemma 1 there exists $\delta>0$ for which we can find subsequences $\left\{x_{n_{k}}\right\}$ and $\left\{x_{m_{k}}\right\}$ of $\left\{x_{n}\right\}$ with $n_{k}>m_{k}>$ ksuch that

$$
\lim _{k \rightarrow \infty} d\left(x_{n_{k}}, x_{m_{k}}\right)=\lim _{k \rightarrow \infty} d\left(x_{n_{k}-1}, x_{m_{k}-1}\right)=\delta
$$

Setting $x=x_{m_{k}-1}$ and $y=x_{n_{k}-1}$ in (1), we obtain

$$
\begin{aligned}
h\left(1,1, \psi\left(d\left(x_{n_{k}}, x_{m_{k}}\right)\right)\right) & \leq h\left(\alpha\left(x_{m_{k}-1}, x_{m_{k}}\right), \alpha\left(x_{n_{k}-1}, x_{n_{k}}\right), \psi\left(d\left(x_{n_{k}}, x_{m_{k}}\right)\right)\right) \\
& \leq \mathscr{F}\left(\beta\left(d\left(x_{m_{k}-1}, x_{n_{k}-1}\right)\right), \psi\left(d\left(x_{m_{k}-1}, x_{n_{k}-1}\right)\right)\right) \\
& \leq \beta\left(d\left(x_{m_{k}-1}, x_{n_{k}-1}\right)\right) \psi\left(d\left(x_{m_{k}-1}, x_{n_{k}-1}\right)\right),
\end{aligned}
$$


which implies that

$$
\frac{\psi\left(d\left(x_{n_{k}}, x_{m_{k}}\right)\right)}{\psi\left(d\left(x_{m_{k}-1}, x_{n_{k}-1}\right)\right)} \leq \beta\left(d\left(x_{m_{k}-1}, x_{n_{k}-1}\right)\right) \leq 1 .
$$

Letting $k \rightarrow \infty$ and using (8) and (6), we obtain

$$
\lim _{k \rightarrow \infty} d\left(x_{n_{k}-1}, x_{m_{k}-1}\right)=0 \leq \delta
$$

which is a contradiction.

This shows that $\left\{x_{n}\right\}$ is a Cauchy sequence and hence is convergent in the complete set $F$. Hence $x_{n} \rightarrow z \in F$ as $n \rightarrow \infty$.

First, we suppose that $T$ is continuous. Therefore, we have

$$
z=\lim _{n \rightarrow \infty} x_{n+1}=\lim _{n \rightarrow \infty} T x_{n}=T \lim _{n \rightarrow \infty} x_{n}=T z .
$$

Next, we suppose that condition (b) holds. Therefore, $\alpha(z, T z) \geq 1$. Now, by (1), we have

$$
\begin{aligned}
h\left(1,1, \psi\left(d\left(T z, x_{n+1}\right)\right)\right) & \leq h\left(\alpha(z, T z), \alpha\left(x_{n}, T x_{n}\right), \psi\left(d\left(T z, x_{n+1}\right)\right)\right) \\
& \leq \mathscr{F}\left(\beta\left(d\left(z, x_{n}\right)\right), \psi\left(d\left(z, x_{n}\right)\right)\right) \\
& \leq \beta\left(d\left(z, x_{n}\right)\right) \psi\left(d\left(z, x_{n}\right)\right),
\end{aligned}
$$

which implies that

$$
\psi\left(d\left(T z, x_{n+1}\right)\right) \leq \beta\left(d\left(z, x_{n}\right)\right) \psi\left(d\left(z, x_{n}\right)\right) .
$$

On taking $n \rightarrow \infty$ and using the properties of $\psi$ and $\beta$, we have $d(T z, z)=0$, that is, $z=T z$.

\section{Some consequences of the main result}

If $h(x, y, z)=(z+l)^{x y}, l>1, \mathscr{F}(x, y)=x y+l, \psi(t)=t$, and $F=X$, in Theorem 2, we have Theorem 4 of [4].

Corollary 1 Let $(X, d)$ be a complete metric space and $T: X \rightarrow X$ be an $\alpha$-admissible mapping. Assume that there exists a function $\beta:[0, \infty) \rightarrow[0,1)$ such that, for any bounded sequence $\left\{t_{n}\right\}$ of positive real,$\beta\left(t_{n}\right) \rightarrow 1$ implies $t_{n} \rightarrow 0$, such that

$$
(d(T x, T y)+l)^{\alpha(x, T x) \alpha(y, T y)}=\beta(d(x, y)) d(x, y)+l
$$

for all $x, y \in X$. Suppose that either

(a) $T$ is continuous, or

(b) if $\left\{x_{n}\right\}$ is a sequence in $X$ such that $x_{n} \rightarrow x, \alpha\left(x_{n}, x_{n+1}\right) \geq 1$ for all $n$, then $\alpha(x, T x)=1$

If there exists $x_{0} \in X$ such that $\alpha\left(x_{0}, T x_{0}\right) \geq 1$, then $T$ has a fixed point. 
If $h(x, y, z)=(x y+l)^{z}, l>1, \mathscr{F}(x, y)=(1+m)^{x y}, m=1, \psi(t)=t$, and $F=X$, in Theorem 2, we have Theorem 6 of [4].

Corollary 2 Let $(X, d)$ be a complete metric space and $T: X \rightarrow X$ be an $\alpha$-admissible mapping. Assume that there exists a function $\beta:[0, \infty) \rightarrow[0,1)$ such that, for any bounded sequence $\left\{t_{n}\right\}$ of positive reals, $\beta\left(t_{n}\right) \rightarrow 1$ implies $t_{n} \rightarrow 0$, such that

$$
(\alpha(x, T x) \alpha(y, T y)+1)^{d(T x, T y)}=2^{\beta(d(x, y)) d(x, y)}
$$

for all $x, y \in X$. Suppose that either

(a) $T$ is continuous, or

(b) if $\left\{x_{n}\right\}$ is a sequence in $X$ such that $x_{n} \rightarrow x, \alpha\left(x_{n}, x_{n+1}\right) \geq 1$ for all $n$, then $\alpha(x, T x)=1$ If there exists $x_{0} \in X$ such that $\alpha\left(x_{0}, T x_{0}\right) \geq 1$, then $T$ has a fixed point.

If $h(x, y, z)=x y z, \mathscr{F}(x, y)=x y, \psi(t)=t$, and $F=X$, in Theorem 2, we have Theorem 8 of [4].

Corollary 3 Let $(X, d)$ be a complete metric space and $T: X \rightarrow X$ be an $\alpha$-admissible mapping. Assume that there exists a function $\beta:[0, \infty) \rightarrow[0,1)$ such that, for any bounded sequence $\left\{t_{n}\right\}$ of positive reals, $\beta\left(t_{n}\right) \rightarrow 1$ implies $t_{n} \rightarrow 0$, such that

$$
\alpha(x, T x) \alpha(y, T y) d(T x, T y)=\beta(d(x, y)) d(x, y)
$$

for all $x, y \in X$. Suppose that either

(a) $T$ is continuous, or

(b) if $\left\{x_{n}\right\}$ is a sequence in $X$ such that $x_{n} \rightarrow x, \alpha\left(x_{n}, x_{n+1}\right) \geq 1$ for all $n$, then $\alpha(x, T x)=1$ If there exists $x_{0} \in X$ such that $\alpha\left(x_{0}, T x_{0}\right) \geq 1$, then $T$ has a fixed point.

Example 3 Let $X=[1, \infty)$ be endowed with the usual metric $d(x, y)=|x-y|$ for all $x, y \in X$ and $T: X \rightarrow X$ be defined by

$$
T(x)= \begin{cases}\frac{x+14}{8} & 1 \leq x \leq 4 \\ \frac{x^{2}}{4}, & x>4\end{cases}
$$

Define the function $\alpha, \beta, \psi$ given by

$$
\begin{aligned}
\alpha(x, y) & = \begin{cases}1, & x, y \in[1,4] \\
0, & \text { otherwise }\end{cases} \\
\beta(t) & =\frac{1}{1+t}, \psi(t)=t
\end{aligned}
$$

Then $T$ is $\alpha$-admissible and we obtain $1 \leq y \leq x \leq 4$

$$
\begin{aligned}
\alpha(x, T x) \alpha(y, T y) d(T x, T y) & =d\left(\frac{x+14}{8}, \frac{y+14}{8}\right)=\frac{1}{8}|x-y| \\
& \leq \beta(d(x, y)) \psi(d(x, y))
\end{aligned}
$$

Hence, $T$ satisfies all the assumptions of Theorem 2 with $H(x, y, z)=x y z$ and $f(s, t)=s t$ and thus it has a fixed point (which is $x=2$ ). 


\section{References}

[1] S. BANACH, Sur les opéerations dans les ensembles abstraits et leur application aux équations integrales, Fund. Math., 3 (1922), 133-181.

[2] A. H.ANSARI,Note on “ $\alpha$-admissible mappings and related fixed point theorems, The 2 nd Regional Conference onMathematics And Applications,PNU,September 2014, 373-376

[3] A. H. Ansari, S. Shukla, Some fixed point theorems for ordered $F$ - $(\mathscr{F}, h)$-contraction and subcontractions in 0-f-orbitally complete partial metric spaces,J. Adv. Math. Stud.Vol. 9(2016), No.1,37-53

[4] N . Hussain, E. KARAPInAR, P. SAlimi, F.AKBAR, $\alpha$-admissible mappings and related fixed point theorems, Journal of Inequalities and Applications 2013.

[5] B. Samet, C. Vetro, P. Vetro, Fixed point theorem for $\alpha-\psi$ contractive type mappings, Nonlinear Anal. 75 (2012), 2154-2165.

[6] J. HARJANi, K. SADARANGANi, Fixed point theorems for weakly contractive mappings in partially ordered sets, Nonlinear Anal. 71 (2009), 3403-3410.

[7] A. Amini-HaRAndi, H. Emami,: A fixed point theorem for contraction type maps in partially ordered metric spaces and application to ordinary differential equations, Nonlinear Anal. 72 (2010), 2238-2242.

[8] J. JACHYMSKI, Equivalent conditions for generalized contractions on (ordered) metric spaces, Nonlinear Anal. 74(2011), 768-774.

[9] B. Samet, C. Vetro, P. Vetro, Fixed point theorem for $\alpha-\psi$ contractive type mappings, Nonlinear Anal. 75 (2012), 2154-2165.

[10] G. V. R. BABU, P. D. SAILAJA, A Fixed Point Theorem of Generalized Weakly Contractive Maps in Orbitally Complete Metric Spaces, Thai Journal of Mathematics, Volume 9 (2011), $1-10$ 